\title{
Perceiving the Center of Three-Body Displays: The Role of Size-Ratio, Symmetry, Elongation, and Gravity
}

\author{
Jay Friedenberg ${ }^{*}, 1$ and Bruce Liby ${ }^{2}$ \\ ${ }^{I}$ Department of Psychology, Manhattan College, Riverdale, New York, USA \\ ${ }^{2}$ Department of Physics, Manhattan College, Riverdale, New York, USA
}

\begin{abstract}
Participants judged the perceived center of three-dot displays at different orientations. In Experiment 1, the dots formed an equilateral triangle. The direction of the response distribution aligned with the largest dot, along axes of reflectional symmetry and with the gravitational down. In Experiment 2, we created isosceles triangles where the distance between one of the dot pairs was varied. Errors were higher for configurations where the symmetry axis was horizontal and a virtual elongation axis was vertical. The results of Experiment 3 replicate this finding and show that response direction is an accommodation to gravity and other shape factors.
\end{abstract}

Keywords: Center of mass, symmetry, elongation.

\section{INTRODUCTION}

The perceived center of an object is the point about which the bulk of the object is seen as balanced or distributed. Mathematically, there are many different ways to compute such a center. For two-dimensional shapes made up of lines, several mathematical centers can be computed corresponding to the barycenter of the vertices, contour, and area [1]. In these figures, the center of gravity or centroid is a geometric center calculated as the mean position of all its points or parts. It can be determined by averaging across the object's $\mathrm{x}$ - and y-coordinates [2]. A centroid is thus the location for which the sum of all distances to each point in the figure is minimal. If the points on the object have equal mass then the term center of mass can be used.

Estimation of a perceptual center serves several functions. It can be used for grasping and manipulation of objects. Participants will grasp wooden planar shapes using tongs at their center of mass [3]. A perceptual center can be calculated as a compact way of representing the location of an object. It could be used to center the origin of a reference frame on a shape [4]. The reference frame could then be used to describe the shape, enabling pattern recognition.

Most center of mass estimation studies have looked at either large single objects or the cloud-like distribution of multiple small objects. In this study, we investigate the intermediate domain of multiple large objects. These stimuli are useful to study for several reasons. They can be manipulated to clarify the influence that size ratio plays in biasing center estimation because the relative size of the dots is easily altered. The number, length, and orientation of symmetry and elongation axes in these stimuli are also easily manipulated allowing for the further investigation of these factors.

*Address correspondence to this author at the Department of Psychology, Manhattan College, Riverdale, New York, USA;

E-mail: jay.friedenberg@manhattan.edu
Center estimation for these types of patterns is useful in a number of real world situations. It is needed when balancing objects on a surface. Imagine for example a serving tray with three drinks located at different points, each with varying amounts of liquid. Visual estimation of the center of mass, independent of haptic feedback, would specify where to place one's hand underneath the tray so that it balances.

There are also some single objects with separated mass distributions, such as a dumbbell, that approximate the distribution of two or more distinct objects. The center of mass can be used for grasping and manipulation of these objects.

The visual system may additionally use the center of mass to denote the location of a group of objects. Tracking group location is helpful in several situations. It could be used, for example, to identify the location of one or more subgroups of people embedded in a larger crowd.

A number of different variables have been found to influence judgment of a perceptual center. The most important of these is symmetry. In one study participants estimated the center of amoeboid, oval, equilateral triangle, square and circle shapes [5]. Variability decreased with an increase in the number of symmetry axes. This result has been replicated using polygonal shapes [6]. Accuracy increased with the number of reflectional symmetry axes. This study found that ability to locate the center was good for shapes possessing only rotational symmetry. It was concluded that rotational symmetry alone appears to be sufficient for accurate center identification, although if reflectional symmetry is present, it will be used.

Another study varied the number of reflective axes and rotational symmetries in single shapes [7]. There was increased accuracy with an increase in the amount of rotational symmetry. When shapes had reflective symmetry, errors were reduced perpendicular to the symmetry axis. When shapes had rotational symmetry, random error in different directions was reduced. The direction of maximum variability in compact two-dimensional shapes has been found to align with the axis of symmetry for shapes having only one symmetry axis [8]. 
A second factor affecting perceptual center estimation is elongation. The distribution of center responses for contour quadrangles is along the main axis of orientation [2]. When these distributions were very elongated, they closely approximated the major orientation axis of the shape. In addition, there has been an increase in center estimation error with an increase in axis length [3].

Elongation is an example of a shape property that carries information about orientation. There are other such cues. For example, in some triangles there is a wide base that tapers toward an apex. Independent of the triangle's actual orientation, this information can cause an observer to judge the base as "down" and the apex as "up". Center estimates have been found to be displaced toward the apex and away from the base in upright equilateral triangles [9]. When the equilateral was rotated, this effect disappeared, suggesting that it was alignment with perceived gravitational vertical that induced the effect. An increase in errors when the major axis of reflectional symmetry was vertical is another result [3]. The response distribution in this case aligned with both the symmetry and perceived gravitational axes.

However, one finding shows a displacement of the perceptual center away from the base in isosceles triangles at every orientation [10]. In these stimuli, there is always a salient base to signal the location of down. An up-down axis can be aligned with the base and apex in these triangles regardless of any perceived gravitational down. One study showed polygonal contour shapes at frontoparallel and horizontal presentation planes. There was no difference in the results, suggesting that gravity was not playing a role [6].

These experiments imply that both intrinsic directional properties such as the base and apex as well as extrinsic directional properties such as gravity can affect the location of the perceived center. When there is no single strong intrinsic directional as is seen in equilateral triangles, gravity can influence judgments. When intrinsic cues are strong as is the case with isosceles triangles and other shapes, the influence of gravity disappears.

In the current study we further investigate the role that size ratio, symmetry, elongation and gravity play in the estimation of a perceived center. Unlike most previous studies, we do not use single shapes. Instead, we have participants judge the center of multi-body displays. Our stimuli consist only of three black-filled dots arranged into different triangular configurations. One advantage of using such patterns is that there are no obvious confounding shape variables like vertices, contours and overall area that are present when single objects are presented.

In previous work using two dot displays, we found that center of mass judgments were affected systematically by dot size ratio and separation [11]. Participant's estimates were remarkably accurate, meaning close to the true center location, except for when the true center fell close to the larger dot's edge, indicating a reluctance to place estimates near or inside one of the dots. In this study, a dimensionality closer to one produced the best fit to the data, suggesting that observers used either diameters or surface area interpretations of the dots when performing their judgments.

There are several aims in the current study. First, we wish to investigate the role size ratio plays in patterns with more than two dots. In our earlier study, responses were always attracted to the larger of two masses [11]. With three dots we can investigate more complicated situations such as when two dots are equal but larger in mass than a third.

Our second aim is to study the influence of symmetry and elongation. In line with previous work, we anticipate that axes of symmetry and elongation will affect the distribution of center estimates, even when there is no visible contour in the pattern. A final aim is to study the role of gravity in center estimation. We anticipate that responses will be pulled downward in the absence of other strong intrinsic shape factors.

In Experiment 1 we present equidistant dots in the form of equilateral triangles at different orientations. We then vary the size ratio of the dots to one another. As in our previous work, we expect that center estimates will be pulled toward larger objects. However, when the dots are all the same size we predict that more global configural forces will be at work. Reflectional symmetry and the perceived up-down axis as determined by gravity should then influence responding.

In Experiment 2 we hold size ratio constant and investigate the spacing between dots. We vary the distance between two of the dots in the expectation that they will group when they are closer together, pulling center estimates toward them. In this study we also vary the length of symmetry and elongation axes. We anticipate that accuracy, defined as distance of the estimates from the true center, will get worse as the length of either of these two kinds of axis increase.

Finally in a third experiment, we vary the orientation of the symmetry and elongation axes. We predict that errors will increase the closer the symmetry axis is to the horizontal and the closer the elongation axis is to the vertical. The results are interpreted according to a process whereby observers use the intersection of the median lines to locate the perceptual center.

\section{EXPERIMENT 1}

\section{Method}

Participants. Twenty-four undergraduate students attending Manhattan College participated voluntarily to obtain extra class credit. There were five males and 19 females with a mean age of 21 years. All participants had normal or corrected-to-normal vision.

Apparatus. The stimuli were three black-filled dots against a white background. The dots were centered on the three points of an invisible equilateral triangle. The center of this virtual triangle was positioned at the center of the computer screen. We varied the relative sizes of the dots in proportion to one another.

The smallest dot measured $3 \mathrm{~mm}$ in diameter and subtended approximately $0.33^{\circ}$ of visual angle. The other dots were either twice as wide or four times as wide as this. The distance separating dot centers was always $65 \mathrm{~mm}$ or about $6.5^{\circ}$ visual angle at a viewing distance of $40 \mathrm{~cm}$.

The relative size of the dots to one another was the first independent variable. We named this variable size ratio. There were seven size ratio conditions. In one grouping, the dots were all the same size, either all the smallest size (des- 
ignated 111), all at the intermediate size of twice the smallest (222), or all at the largest size (444). In a second grouping, two dots had a greater diameter than the third. They could be twice (122) or four times (144) in diameter. A final grouping had one dot either two times (112) or four times (114) larger than the others. Fig. (1) shows these conditions that we named "Same-size", "Two-big" and "Two-small", respectively.

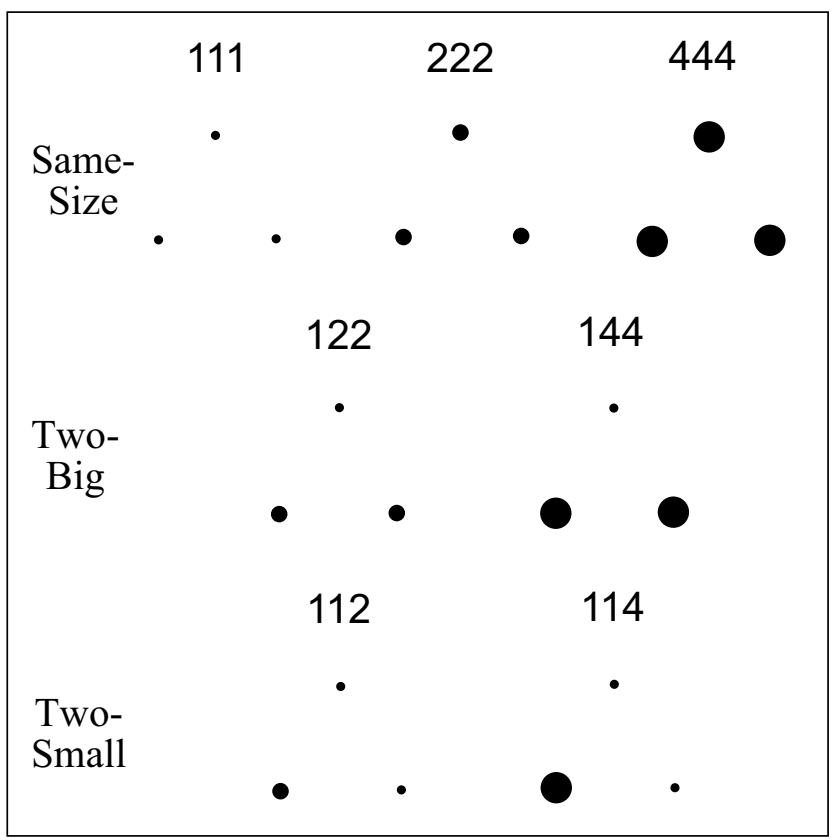

Fig. (1). The three sets of stimuli in Experiment 1. Dots were either all the same size $(111,222,444)$, or two dots shared the same larger $(122,144)$ or smaller $(112,114)$ size. Ratios were twice or four times the smallest dot.

Differing sizes were assigned to dots clockwise from a default upright orientation where one of the triangle's apices pointed upwards. In the two-big condition (122 and 144), the topmost dot was smallest. In the two-small condition (112, 114) the bottom-left dot was largest.

Our second independent variable was orientation. Each size ratio condition was presented at eight different orientations, starting at the vertical of the default orientation $\left(0^{\circ}\right)$ and continuing in $45^{\circ}$ increments clockwise. In order, these orientations were $0^{\circ}, 45^{\circ}, 90^{\circ}, 135^{\circ}, 180^{\circ}, 225^{\circ}, 270^{\circ}$, and $315^{\circ}$.

Procedure and Design. Participants signed a consent form and read instructions for the task. The instructions asked them to estimate the center-of-mass. This was described as a point of balance or equilibrium. They were shown an example of one of the stimuli, with the actual center indicated by a crosshair.

On each trial the stimulus appeared along with a small black positioning dot. Using the mouse, the participants moved this dot to what they thought was the perceived center. When they were satisfied with their judgment they pushed the space bar to end the current trial. The positioning dot was smaller than the smallest dot of the triangular configuration and appeared well outside of it in random locations on each trial. This was done to insure that it did not interfere with participant's estimates.
A block of 56 trials consisted of the combination of seven size ratios at eight orientations. Participants ran through three blocks, completing a total of 168 trials. When finished, participants filled out an information form and read a debrief explaining the purpose of the study. Each experimental session lasted about one-half hour.

\section{Results and Discussion}

The data were first screened for outliers. This was based on reaction time, measured in seconds. All responses that took longer than 25 seconds were considered outliers and removed from the analyses. They constituted far less than one percent of the data. These long responses were considered overly analytical. Most participants responded quickly. Average reaction time with outliers removed was only 6.26 seconds. There was no clear pattern of result with reaction time so it was not considered a dependent variable.

The center of mass of the three body system was calculated in the following, standard way. Each of the darkened circles represents a uniform, spherical body. As these were identical, equal masses for all three was assumed. A twodimensional representation is presented to the participant, thus the center of mass, when plotted on an X-Y graph, will have two coordinates. These are calculated with:

$$
\begin{aligned}
X_{\text {COM }} & =\frac{M_{1} X_{1}+M_{2} X_{2}+M_{3} X_{3}}{M_{1}+M_{2}+M_{3}} \\
Y_{\text {СOM }} & =\frac{M_{1} Y_{1}+M_{2} Y_{2}+M_{3} Y_{3}}{M_{1}+M_{2}+M_{3}}
\end{aligned}
$$

where the subscripts 1,2 , and 3 refer to the particular darkened circle. Since the masses are assumed to be equal, this reduces to

$$
\begin{gathered}
X_{\text {СОM }}=\frac{X_{1}+X_{2}+X_{3}}{3} \\
Y_{\text {СOM }}=\frac{Y_{1}+Y_{2}+Y_{3}}{3}
\end{gathered}
$$

The positions of the darkened circles are indicated by the coordinates of their centers. The computer software has its own unit of measure. The lower left hand corner of the monitor screen is at coordinate $(0,0)$; all measured and calculated positions are made with respect to that position.

The triangular arrangement of the three body system located at different positions on the screen, depending on condition. The dependent variables error and direction are measured with respect to the actual position of the center of mass for each orientation and geometry.

Error Analyses. A (7x8) factorial analysis of variance (ANOVA) with size ratio and orientation as factors was performed. Error was the dependent measure and was calculated as the Euclidean distance between the response and the true center-of-mass. Error values are reported in millimeters. There was a significant main effect of size ratio $[F(6,1,288)$ $=751.3, \mathrm{p}<.01]$, of orientation $[\mathrm{F}(7,1,288)=18.23, \mathrm{p}<$ $.01]$ and of their interaction $[\mathrm{F}(42,1,288)=4.1, \mathrm{p}<.01]$. Fig. (2) shows mean errors with \pm 1 standard error for the interaction. 


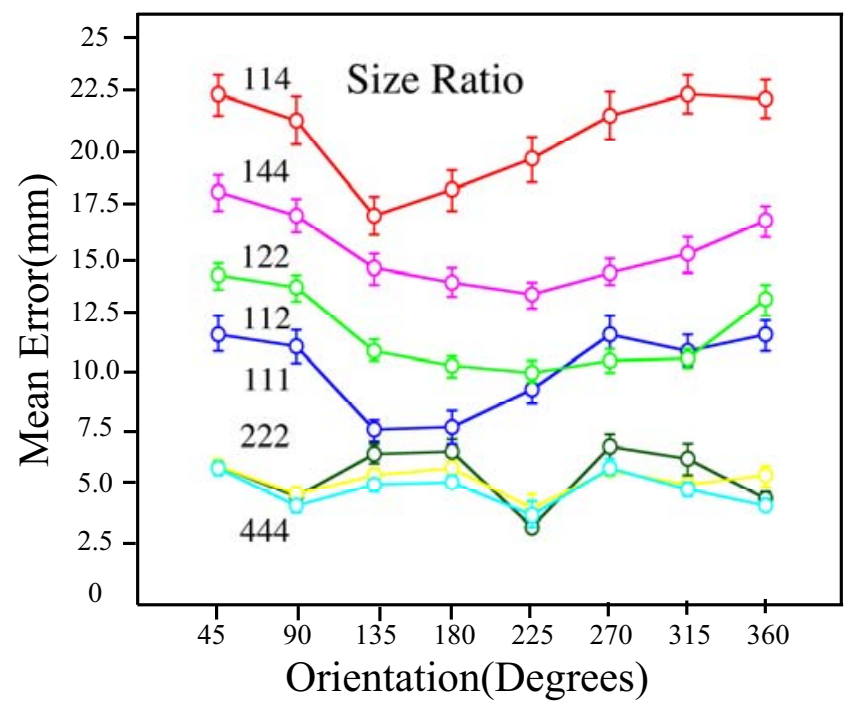

Fig. (2). Mean error for each size ratio and orientation in Experiment 1 . Error was the difference between estimates and the location of the true center. Bars indicate $\pm 1 \mathrm{~S}$. E.

Accuracy was greatest in the same-size conditions. In these cases center-of-mass estimation is equivalent to locating the center of the triangle. There is abundant information available to locate this center, as it is the intersection of the triangle's three axes of reflectional symmetry. Accuracy improved slightly with an increase in dot size. The 444 case had the fewest errors, the 222 case had slightly more, and the 111 case had somewhat more. However, these differences are small as judged by the degree of overlap between the three equal dot size curves in Fig. (2). This kind of effect makes sense though, as bigger dots are closer to the center of the triangle. The shorter the distance between the dots and the center, the easier estimation should be.

The error functions for each subset of size ratio are similar. For the same-size conditions there is a large dip in the functions at $180^{\circ}$ and smaller dips at $45^{\circ}$ and $315^{\circ}$. For the two-small cases the functions dip at $90^{\circ}$. Here, error is greater for 114 than for 112 . This is because the larger dot exerts more of an influence, biasing responses closer to it. For the two-big cases the functions are nearly identical with errors again greater for the larger dots.

Direction Analyses. Next, another factorial ANOVA with size ratio and orientation was performed. This time, the direction of the response distribution served as the dependent variable. Direction refers to the angular orientation of a response from the true center-of-mass. Mean direction indicates which way the response distribution is being pulled or stretched. A mass larger than those around it ought to pull the bulk of responses outward from the center of the display toward itself. Direction was calculated as the orientation of a line drawn from the true center to a response. It was measured in the same way as the orientation variable, as angular deviation from the vertical.

One problem with analyzing response direction is that there may be more than one mode. In other words, responses can exist at more than a single orientation. When this is the case, averaging the directions can be misleading. For example, if responses are equally distributed at $90^{\circ}$ and $180^{\circ}$, the mean is $135^{\circ}$ and not indicative of perceived pointing. For this reason, we examined the distribution of perceived direction. For each stimulus there was only a single peak in the distribution with little difference between measures of central tendency. We therefore analyzed these data using standard statistical procedures, i.e., without using circular statistical techniques.

There was a significant main effect of size ratio $[\mathrm{F}(6$, $1,288)=2.9, \mathrm{p}<.05]$ and orientation $[\mathrm{F}\{7,1,288)=151.8, \mathrm{p}$ $<.01]$. The interaction was also significant $[F(42,1,288)=$ $22.1, \mathrm{p}<.01]$. We discuss the results once more in terms of the three subgroups. For the two-small condition, the arm of the response distribution always pointed toward the larger dot. Here, size ratio is the effective influence on direction, with the larger dot pulling responses toward it. Panel (A) of

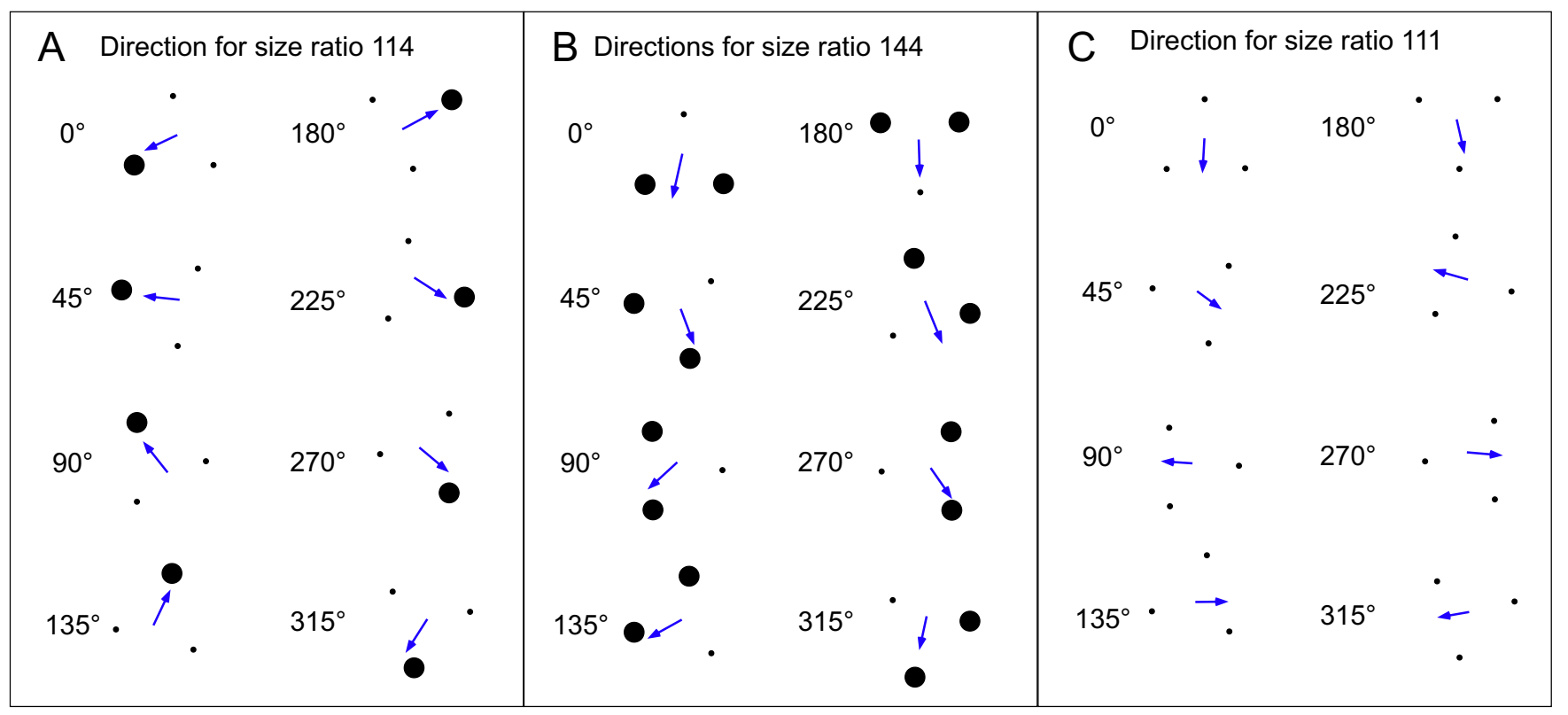

Fig. (3). The arrow indicates the mean direction of the response distribution for three selected conditions in Experiment 1. Panel A shows the Two-Small condition, panel B shows the Two-big condition and panel C shows the Same-Size condition. 
Fig. (3) graphically depicts the mean direction for each orientation of the 114 condition.

In the two-big conditions there is another factor affecting the results: the downward pull of gravity. Downward means toward the bottom of the screen, since the stimuli were presented here and in the other experiments in a frontoparallel fashion where the screen surface was perpendicular to the direction of gaze. The mean response distribution for these cases always pointed within $45^{\circ}$ clockwise or counterclockwise of straight down $\left(180^{\circ}\right)$, indicating a perceived gravitational influence that tugged responses in this direction. If one of the two larger dots was near the down position, responses pointed to it, indicating that size ratio could only compete with gravity when it was close by. Panel (B) of Fig. (3) shows mean direction for the 144 condition.

In the same-size group we see yet a third force at work: symmetry. Direction was always aligned near one of the triangle's three reflectional symmetry axes. When the symmetry axis was vertical, as in the $0^{\circ}$ and $180^{\circ}$ cases, it pointed downward, indicating a gravitational influence. In all other cases, responses aligned themselves along the symmetry axis closest to a horizontal orientation. It is important to note that the symmetry axes in these displays is not for an individual shape. It is that formed by the collection of all three objects taken together.

Direction in this group always pointed away from the single dot near the horizontal. For example, in the $90^{\circ}$ case, the triangle "points" to the right and responses are pulled to the left. In the $270^{\circ}$ case, these directions are reversed. This pointing effect is perhaps a bias toward the perceived "base" of the triangle, the base being the direction flanked by two dots rather than the bottom. The two dots, being aligned at or near vertical, may be perceived as the more stable end of the pattern. Panel (C) in Fig. (3) shows mean direction for the 111 condition.

Three forces can thus nicely account for the results of this experiment. These forces can be categorized as either extrinsic or intrinsic. Gravity is an extrinsic force because it applies independently of the pattern. Because the stimuli were viewed on a frontoparallel screen surface, gravity would always be down regardless of the particular characteristics of any dot pattern. On the other hand, size ratio and symmetry are intrinsic because they do depend on the stimulus, resulting from either differences in dot sizes or in the number and orientation of the symmetry axes.

In the two-small patterns where one dot was larger than the other two, size ratio overwhelms gravity and responses point to the larger dot. In these stimuli, the responses also fall along the symmetry axis, so symmetry reinforces size differences instead of competing with it. In the two-big conditions gravity appears to reinforce one size ratio alternative, since the responses could have pointed to either of the two large dots equally often. Instead, they always pointed downward or to the largest dot closest to down. Symmetry only plays a role here when it is vertical.

In the same-size condition where there are no size differences, symmetry appears to rule and pointing is always along the vertical or horizontal symmetry axis. Gravity again influences, but only when it reinforces symmetry. The literature on symmetry perception typically shows that vertical sym- metry is the most salient, followed by horizontal and then oblique symmetry [13]. In our patterns, responses rarely aligned along an obliquely oriented symmetry axis.

\section{EXPERIMENT 2}

With equilateral triangles, distance between dots is constant and cannot influence judgments. However, dot separation does affect center-of-mass estimation. In previous work, we found strong distance effects for two dot patterns [11]. As separation between two dots of differing sizes decreases, estimates move correspondingly closer to the larger dot, tracking changes in the true center position. In addition, we found that accuracy improved when the two dots were closer to one another, but not so close that the true center fell on or near the larger dot's edge.

One consequence of moving dots closer together is that they may form a perceptual group. According to the gestalt principle of proximity, the closer two items are, the more strongly they will group assuming all other factors such as similarity are equal [12]. Grouping by proximity could play an important role in center-of-mass estimation. Proximal dots might be perceived as having a combined mass greater than their actual physical masses. They could pool their effects, causing observers to estimate the center closer to them than it really is.

To see if this is the case, we performed a second experiment using isosceles triangles, those having two equal sides. We manipulated the distance of one "side" while holding the length of the other two "sides" constant. If there is a perceptual grouping effect, we should see center-of-mass estimates move closer to the two proximal dots. Alternatively, it may be that local grouping factors are overshadowed by larger global properties of the stimuli, such as symmetry or orientation, in which case we may see little or no grouping effects.

\section{Method}

Participants. Sixteen undergraduate students from Manhattan College participated to fulfill an extra credit assignment. There were six males and ten females. Mean age was 20 years. Vision was normal or corrected-to-normal.

Apparatus. It is important to note that in all of the experiments reported here, none of the sides of the triangles were actually visible. They existed only in terms of the relationships between dots that were visible. We created differently shaped isosceles "triangles" formed by distances between dots. In each, the length of one side was varied while the length of the other two sides remained the same. This served as the first of our independent variables.

We began with a canonical upright isosceles triangle. The three dots forming the apices of the triangle we labeled as A, $\mathrm{B}$ and $\mathrm{C}$, starting with the "top" dot and proceeding clockwise. The two distances $\mathrm{AB}$ and $\mathrm{AC}$ were the same for all stimuli in the experiment. We varied the distance BC by changing the angle at dot $\mathrm{A}$. When this angle is increased, distance BC also increases. Fig. (4) indicates these dots and the angle in the $80^{\circ}$ condition.

We produced five different shapes corresponding to angles of $20^{\circ}, 40^{\circ}, 80^{\circ}, 100^{\circ}$ and $120^{\circ}$. The equilateral case of $60^{\circ}$ was omitted because it was used in the previous experi- 
ment. We substitute the value obtained there in the current results for purposes of comparison.

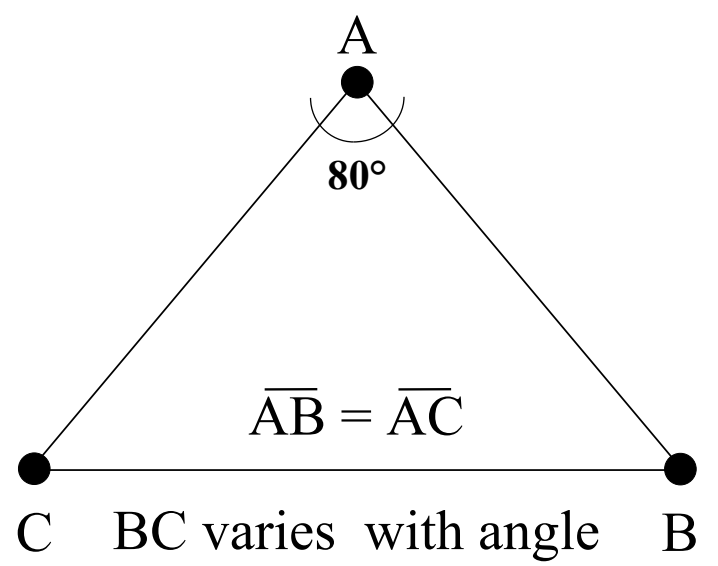

Fig. (4). The $80^{\circ}$ shape condition from Experiment 2. The distance between dots $\mathrm{A}$ and $\mathrm{B}$ and between dots $\mathrm{A}$ and $\mathrm{C}$ was constant for all stimuli. The distance between dots $\mathrm{B}$ and $\mathrm{C}$ co-varied with angle. Sides are shown for ease of interpretation but were not present in the stimuli.

All dots were black and viewed against a white background. The diameter of the dots was $3 \mathrm{~mm}$, the same as the smallest size from Experiment 1. The lengths of $\mathrm{AB}$ and $\mathrm{AC}$ were $50 \mathrm{~mm}$ and subtended approximately $5^{\circ}$ of visual angle. The lengths of $\mathrm{BC}$ for each of the angle measures, going from $20^{\circ}$ through $120^{\circ}$ were $15 \mathrm{~mm}, 30 \mathrm{~mm}, 55 \mathrm{~mm}, 70 \mathrm{~mm}$ and $80 \mathrm{~mm}$ and subtended approximately from $1.5^{\circ}$ to $8^{\circ}$ visual angle. The sides of the "triangle" were never actually visible.

Each of the five shape conditions was presented at four orientations: up $\left(0^{\circ}\right)$, down $\left(180^{\circ}\right)$, left $\left(270^{\circ}\right)$ and right $\left(90^{\circ}\right)$. Orientation in this case corresponds to the orientation of the triangle's symmetry axis in the direction that it bisects the single apex dot. Orientation served as our second major independent variable. Fig. (4) shows the $80^{\circ}$ condition in the upright orientation with sides added for ease of interpretation.

Procedure and Design. The procedure was identical to that of the first experiment. Informed consent was obtained from all participants. They were first instructed on what a center-of-mass is and shown an example of one of the stimuli. They recorded responses by moving the small positioning dot into place and recorded their response by hitting the space bar. There were five shapes combined with four orientations to yield a total of 20 unique stimulus trials in a block. Participants ran through 16 blocks completing a total of 320 trials. An experimental session lasted approximately $40 \mathrm{~min}-$ utes.

\section{Results and Discussion}

Error Analyses. Error was the first dependent measure. It was the distance between the estimate and the actual center-of-mass and was used to indicate task difficulty. There were no major outliers so all data were included in the analyses. We performed a $5 \times 4$ factorial ANOVA with shape and orientation as factors. There was a significant main effect of shape $[\mathrm{F}(4,300)=36.9, \mathrm{p}<.01]$ and orientation $[\mathrm{F}(3,300]=$ $21.2, \mathrm{p}<.01]$. The interaction between them was also sig- nificant $[F(12,300)=3.4, p<.01]$. Fig. (5) shows error for each shape-orientation combination.

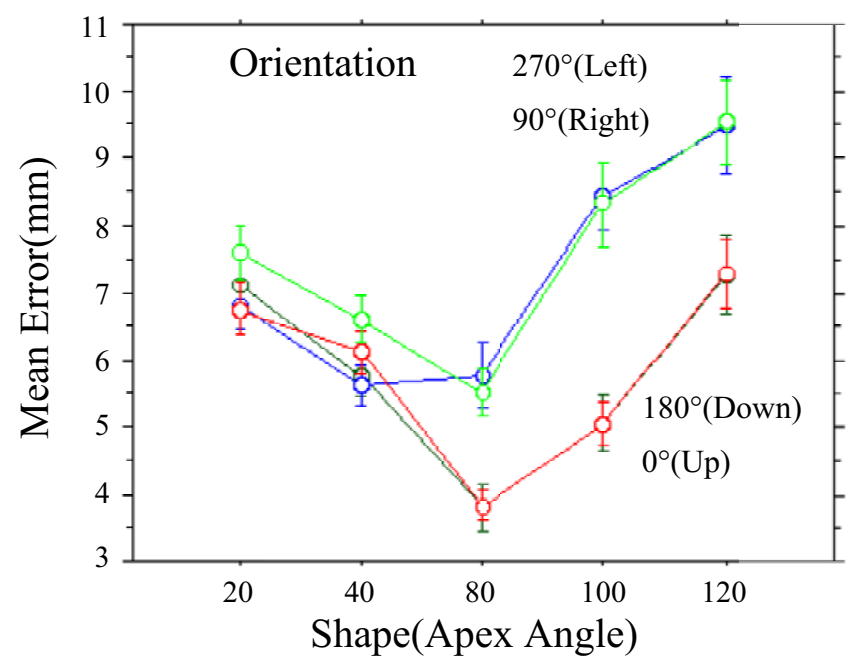

Fig. (5). Mean error for each shape and orientation in Experiment 2. Bars indicate $\pm 1 \mathrm{~S}$. E.

Average error for the 111 condition in Experiment 1 was the equivalent of the $60^{\circ}$ case in this study and was about 3 $\mathrm{mm}$. If we superimpose this value onto the plot in Fig. (5) inbetween $40^{\circ}$ and $80^{\circ}$ we see that errors increased linearly on either side of $60^{\circ}$. Errors increase monotonically as the shape of the triangle deviated away from an equilateral norm. This was the case for shapes with angles both less than and greater than $60^{\circ}$.

The center-of-mass for triangular shapes with equal-sized dots is at the intersection of the median lines. A median is a line extending from each vertex to the midpoint of the opposite side. Hence, one possible way of estimating the center in these stimuli would be to perceptually complete the sides of the triangle, then fill in the medians, and then estimate their intersection. For equilateral triangles, this process would be easy because all sides are equal, all medians are equal and all the medians are symmetry axes. For isosceles triangles it ought to be more difficult since only two of the sides are equal and only one of the medians is a symmetry axis.

If it were true that differing median lengths make it more difficult to estimate their intersection and hence the centerof-mass, then the pattern of error results we obtain make sense. As angle decreases for values less than $60^{\circ}$, the symmetry axis median becomes longer in proportion to the two other medians. As angle increases for values greater than $60^{\circ}$, the symmetry median becomes shorter in proportion to the remaining two medians.

Errors were low for vertical orientations when the triangle pointed up or down. In fact, the difference between these two conditions is indistinguishable in Fig. (5). Errors were much higher for horizontal orientations when the triangle pointed left or right. As stated earlier, it is a well-established finding that vertical symmetry is easier to detect than horizontal symmetry. Our results parallel this finding and suggest that symmetry detection aids in the localization of the center-of-mass. Detecting the symmetry axes in these figures provides one of the three medians and would thereby constrain the position of the center and improve accuracy. 
The interaction between shape and orientation can be explained in terms of an elongation axis, which is the longest axis that fits through a shape. In our stimuli, the elongation axis is virtual. It can be inferred as a line connecting the two most distant dots when apex angle is greater than $60^{\circ}$ and is equivalent to the symmetry axis when apex angle is less than $60^{\circ}$. In Fig. (5), one can see that the performance difference between vertical and horizontal is minimal when angle is less than $60^{\circ}$. The lines connecting mean errors for all four orientations are close together. For these conditions, the symmetry axis and the elongation axis coincide. This may make symmetry more salient at horizontal orientations, perhaps facilitating estimation for those conditions.

In contrast, the performance difference between vertical and horizontal symmetry axis orientations is more pronounced when angle is greater than $60^{\circ}$. Here, the error functions for these two sets of orientations are widely separated. For these conditions the symmetry axis is perpendicular to and shorter than the elongation axis. This may reduce the salience of horizontal symmetry, making estimation for those conditions more difficult.

Direction Analyses. There was no evidence for directions pointing to more than one orientation so mean direction was taken to be a valid measure of perceived pointing. We next performed a factorial ANOVA using direction of the response distribution as the dependent measure. As before, shape and orientation were the two factors. There was no main effect of shape. There was a main effect for orientation $[\mathrm{F}(3,300)=4.3, \mathrm{p}<.01]$ and for the shape by orientation interaction $[\mathrm{F}(12,300)=13.5, \mathrm{p}<.01]$. Fig. (6) shows the interaction.

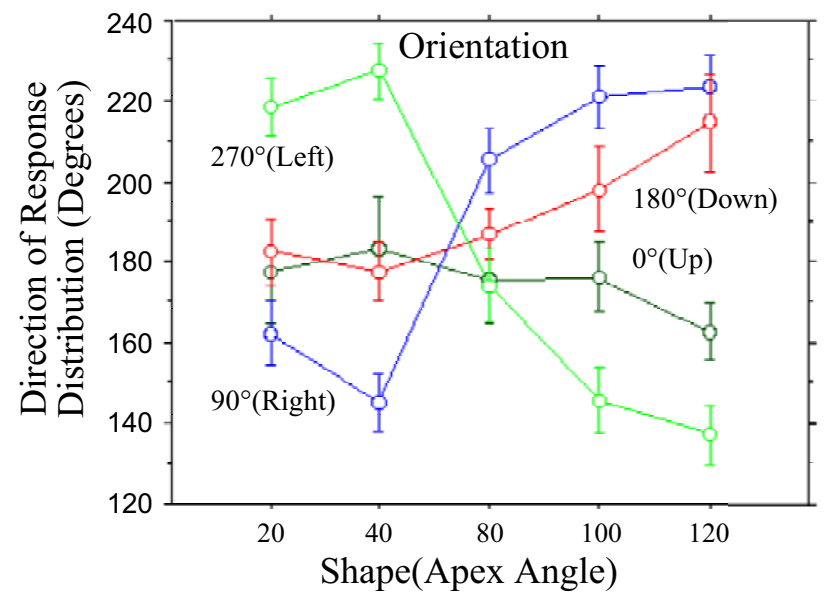

Fig. (6). Mean direction of the response distribution for each shape and orientation in Experiment 2. Bars indicate $\pm 1 \mathrm{~S}$. E.

The fact that there was no main shape effect becomes immediately apparent when one looks at Fig. (6). The direction functions for up and down and for left and right are opposites and cancel each other out. Why should this be? Let us examine the up and down cases first.

For vertical shapes where angle is less than $60^{\circ}$ responses align along the symmetry axis median and point downward. In these cases the symmetry median exerts a powerful influence because it is the longest median and aligns with the gravitational axis. When angle is greater than $60^{\circ}$ the other non-symmetric medians bias estimation as they get longer in proportion to the symmetry median. For the upward pointing triangle $\left(0^{\circ}\right)$, direction rotates counterclockwise, tracking median B (the median intersecting the midpoint of the angle at dot B). For the downward pointing triangle $\left(180^{\circ}\right)$, direction rotates clockwise, tracking median $\mathrm{C}$ (the median intersecting the midpoint of the angle at dot C). Medians B and C are both equidistant from the vertical or gravitational orientation, so these trends seem to represent inherent directional biases in the participants.

For horizontally oriented triangles the story is similar. For right pointing triangles $\left(90^{\circ}\right)$ when angle is less than $60^{\circ}$, direction tracks median $\mathrm{C}$. When angle is greater than $60^{\circ}$ direction tracks median B. For left pointing triangles $\left(270^{\circ}\right)$ when angle is less than $60^{\circ}$, direction tracks median $\mathrm{B}$. When angle is greater than $60^{\circ}$ direction tracks median $\mathrm{C}$.

The results of this experiment fail to support the grouping by proximity hypothesis. As angle decreased, the distance between dots $\mathrm{B}$ and $\mathrm{C}$ diminished causing them to group more strongly by proximity. According to the hypothesis this ought to have pulled the response distribution closer to them. This did not happen. Take for example the right pointing triangle. A decrease in angle ought to have pulled the responses clockwise, leftward in this case, toward the converging dots. Instead the actual distribution swung counterclockwise as angle increased.

What we see here instead is dominance of the global features of the dot configuration. Symmetry and median lines, which did impact on estimates, are global, configural properties of these patterns and can only be formed by taking into account all three dots together. These factors override the more local effect of grouping, which is induced by just a portion of the configuration.

There is an alternate interpretation of this data. With triangular shapes center of mass estimation has been found to correspond more closely to the center of an inscribed circle than to the true center [8]. In order to test whether our participants were also making use of an inscribed circle, we calculated the mean center values for each of our triangular patterns and compared them against the true center location. If participants were using the inscribed circle, their estimates would move toward the base as the triangles became more acute and toward the apex as the triangles became more obtuse. The observed pattern was in fact the opposite of this. We conclude that our participants did not use this strategy, perhaps because it may be difficult to imagine an inscribed circle without any external contours.

\section{EXPERIMENT 3}

In Experiment 2, we saw new influences at work. Elongation in addition to symmetry was found to affect center judgments. As the elongation axis increased in length beyond that of the symmetry axis, accuracy steadily diminished. This effect was particularly pronounced for left and right facing triangles where the axis of elongation was vertical.

It seems that elongation and symmetry both vie for influence over center judgments but that in most cases symmetry wins out. Elongation seems to play a role when it is prominent, i.e., longer than any other axis in the pattern, and when 
it is vertical. In this experiment we set out to further investigate the conditions under which elongation might affect center estimates. We chose the $120^{\circ}$ shape from Experiment 2 because this angle produces a very long elongation axis perpendicular to symmetry. We then presented this shape at various orientations both clockwise and counterclockwise away from the symmetric horizontal. These orientations allow us to study the influence of symmetry and elongation as they deviate about the vertical and horizontal.

In particular, we can investigate how these factors bias both the magnitude of error and the direction of response. Given the results of the previous study, we expect error rates to be highest when elongation and symmetry compete with one another, that is, when elongation is reinforced by alignment near vertical. Accuracy should be better when the symmetry axis is reinforced near vertical.

Response direction should also be quite informative. If symmetry always wins out, direction should align with the symmetry axis. If elongation wins out, direction should instead align with the orientation of the elongation axis, or perhaps along one of the non-symmetry medians approximately parallel to it. A third possibility, and one that seems more likely, is that both symmetry and elongation will influence responses to varying degrees depending on their orientation.

\section{Method}

Participants. Twenty undergraduate students attending Manhattan College participated to obtain extra class credit. There were 15 females and five males. Mean age was twenty years. Vision was normal or corrected-to-normal.

Apparatus. The $120^{\circ}$ triangular shape from Experiment 2 was presented at 16 different orientations. Each orientation corresponded to a $20^{\circ}, 40^{\circ}, 60^{\circ}$ and $80^{\circ}$ rotation of the shape clockwise and counterclockwise from a left and right facing horizontal symmetry axis orientation. This yielded the following symmetry axis orientations: $10^{\circ}, 30^{\circ}, 50^{\circ}, 70^{\circ}, 110^{\circ}$, $130^{\circ}, 150^{\circ}, 170^{\circ}, 190^{\circ}, 210^{\circ}, 230^{\circ}, 250^{\circ}, 290^{\circ}, 310^{\circ}, 330^{\circ}$ and $350^{\circ}$.

Procedure. The procedure was identical to that of the previous two studies. Informed consent was obtained from al participants.

\section{Results and Discussion}

The data were first screened for outliers. Any x- or ycoordinate more than $45 \mathrm{~mm}$ from the true center were omitted from the analyses. These constituted about $1.7 \%$ of total responses.

Error Analyses. A one-way ANOVA with symmetry axis orientation as the factor and error as the dependent variable was performed. There was a significant effect of orientation $[F(15,304)=2.4, p<.01]$. Fig. (7) depicts the means and standard errors. The trend in this figure is straightforward. Accuracy is greatest at symmetry axis orientations near vertical and least for those near horizontal. This effect is gradual and continuous, since errors bottom out near the vertical and gradually rise as the shape rotates toward the horizontal. The effect is stronger for right facing triangles, which may again reflect inherent observer biases. This result replicates our finding from Experiment 2 and supports our hy- pothesis that error rates will be higher when elongation and symmetry compete, i.e., when both are perceptually salient. This is the case when symmetry is horizontal and elongation is vertical.

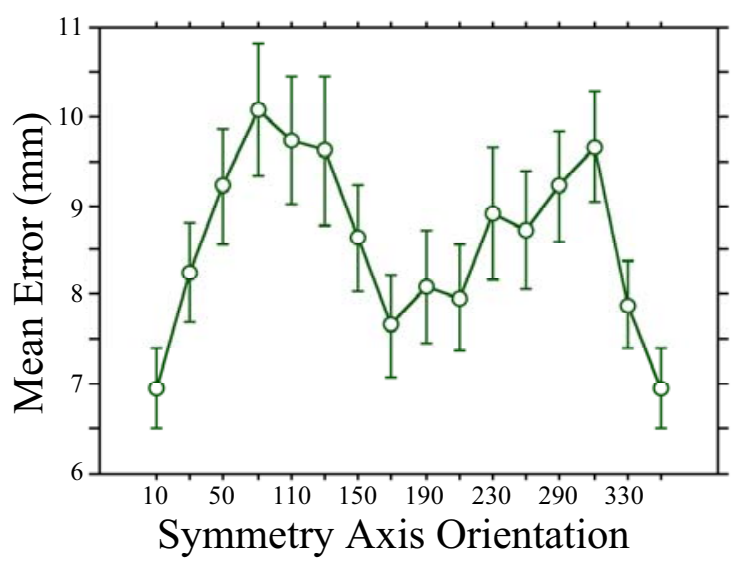

Fig. (7). Mean error as a function of symmetry axis orientation for Experiment 3. Bars indicate \pm 1 S. E.

Direction Analyses. The histograms failed to show evidence of pointing to more than a single orientation so another one-way ANOVA with symmetry axis orientation was performed, this time with direction as the dependent measure. The test yielded a significant result $[F(15,304)=21.6, p$ $<.01$. In order to better interpret the results, we present the angular difference between direction and three different axes in Fig. (8). These are the gravitational axis, which is always pointing downwards, the symmetry axis and the elongation axis. The elongation axis is always perpendicular to symmetry.

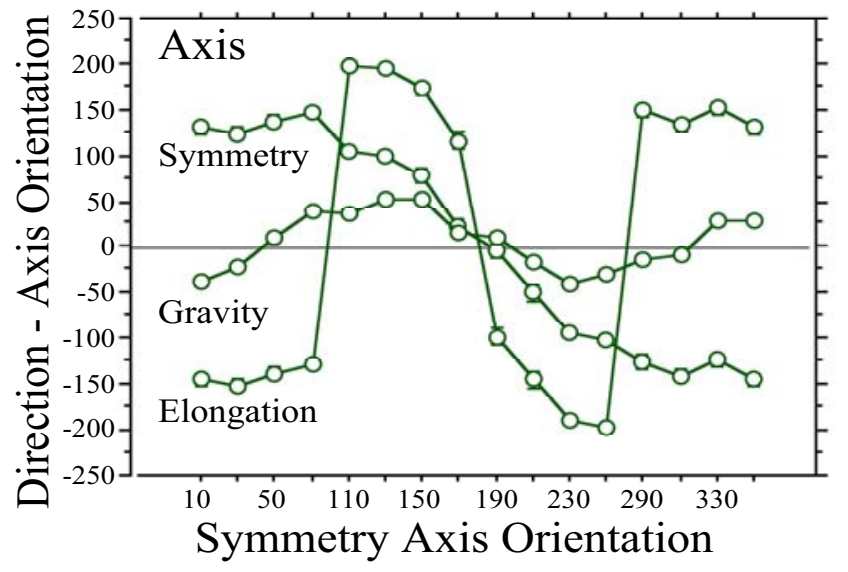

Fig. (8). The difference between direction of the response distribution and the symmetry, gravitational and elongation axes as a function of symmetry axis orientation in Experiment 3. Difference is calculated as (direction - axis orientation). Bars indicate $\pm 1 \mathrm{~S}$. E.

An examination of Fig. (8) reveals several interesting conclusions. First, the responses were most closely aligned with the gravitational axis, or $180^{\circ}$. The difference in orientation between the responses and straight down was smallest. After that they were next most influenced by the symmetry 
axis and finally by the elongation axis. Gravity exerts its greatest influence when the triangle is pointing near down. In this condition, the apex dot is almost at $180^{\circ}$ and may tug responses toward it. Symmetry is also a strong influence for the orientations near down because it is reinforced by gravity. On the other hand, elongation at this orientation has little effect, presumably because it is aligned near the horizontal.

The influence of all three factors diminishes with deviations away from downward either clockwise or counterclockwise to the horizontal orientations near $90^{\circ}$ and $270^{\circ}$. In these near left or right facing directions, the deviation from gravity is large. Symmetry is weak here, perhaps because it aligns at the horizontal, but so is elongation, even though it is vertical.

Deviation from gravity is close to zero at $50^{\circ}$ and $310^{\circ}$. These appear to be "breakpoints" where elongation changes from horizontal to vertical. Perhaps elongation serves as a reference frame at non-diagonal orientations where it is more vertical or horizontal. When it is near diagonal, this reference is lost and participants default back to a gravitational frame.

All in all, these data suggest that no single factor determines the direction of the response distribution. Instead, it is an accommodation between various influences. In some cases, these influences reinforce one another, as is the case with gravity, symmetry and the apex dot near $180^{\circ}$. In other cases, there is competition. For example, at $290^{\circ}$ direction is in-between gravity and symmetry but closer to gravity, reflecting gravity's stronger influence at this orientation.

\section{GENERAL DISCUSSION}

In these experiments we investigated estimation of a perceptual center in multi-body displays. The use of this type of configuration has allowed us to manipulate a number of factors including relative dot size and the number, length, and orientation of symmetry and elongation axes. It has extended our previous findings with size ratio to patterns with more than two dots [11].

Let us now summarize and discuss the specific results of each experiment. In Experiment 1, the dots formed an equilateral triangle presented at various orientations. The relative sizes of the dots were varied. The results were accounted for by three different factors: size ratio, symmetry, and the perceived up-down axis produced by gravity.

When one of the dots was larger than the other two estimates were pulled toward the larger dot. In these patterns there is a pure mass effect. In contrast, when all the dots were the same size, mass played no role. In this case, the response distribution aligned along one of the display's three symmetry axes. When two of the dots were larger than the third, responses were tugged downward toward the gravitational bottom of the pattern, pointing either straight down or toward the largest dot located closest to down.

These results demonstrate an inter-play between intrinsic and extrinsic influences. When the pattern contains noticeable size differences or obvious symmetry, these two intrinsic factors affect center location. When these properties are less salient, the orientation of the display relative to a perceived external gravitational frame of reference best accounts for the results.
Several previous studies have found a "base effect" where responses point toward the perceived bottom or base of a single triangle with visible contours $[10,9]$. This was true in two cases. First, for upright equilaterals where gravity reinforced one of the three sides as downward. It was also found for isosceles triangles with a salient base regardless of orientation.

We failed to replicate this effect. In the two-large condition of Experiment 1 with equilaterals, the two larger dots should form a strong perceptual base. Yet, responses only pointed toward them in the $0^{\circ}$ condition. In all other cases, they pointed to gravitational down. Apparently, size similarity by itself is not enough to induce perception of a base. We also failed to obtain a base effect in Experiment 2. Direction of the response distribution in these isosceles triangles was roughly toward $180^{\circ}$ for upward and downward pointing triangles only. The base effect may require the presence of a visible contour.

In Experiment 2 we employed dots in an isosceles configuration. Errors were lower for vertical and higher for horizontal orientations. This finding follows a similar effect found for symmetry detection [13]. It implies that symmetry detection is part of computing a perceptual center. Because a symmetry axis is a median and the intersection of the medians yields the center, it make sense that observers might first detect the presence of symmetry and use it to judge center location.

It is interesting to speculate on how observers might compute a perceptual center. For single shapes viewed in isolation there are several possibilities. Perceptual center estimates for triangles and quadrilaterals have been found to coincide more closely with an inscribed circle than with the true center-of-mass [8]. The center of the inscribed circle is the point that is equidistant from each of the sides of the polygon. However, these authors note that the inscribed circle can only be used for compact shapes, not those such as a banana or dumbbell. The inscribed circle is just a geometric way of describing how a perceptual center may be computed and does not necessarily indicate the stimulus cues used or process by which observers perform this operation.

In triangles, the center of mass coincides with the average position of the three vertices and with the intersection of the three medians. In these shapes, participants may "trace" along the axes to their intersection [6]. The fact that estimates fall along these axes and are minimized perpendicular to them supports this notion. The center of shapes with rotational symmetry also lies at the focus of radial symmetry. For radial patterns, participants may imagine the shape rotating about the center to superimpose upon itself and place the center at this point of rotation.

As noted earlier, elongation is another property affecting estimation of a perceptual center. Previous work finds that errors increase with an increase in elongation [3]. We also obtained results that can be interpreted according to elongation, although in our case elongation is an inferred property. In our second experiment, errors were higher when an elongation axis formed by connecting the two farthest dots was vertical (the $90^{\circ}$ and $270^{\circ}$ orientations). At these orientations, vertical reinforces elongation, while symmetry is at its less preferred horizontal orientation. The elongation axis 
seems to interfere with detecting symmetry, thereby producing more errors. In comparison, when symmetry does align with vertical (the $0^{\circ}$ and $180^{\circ}$ orientations) errors are lower, ostensibly because vertical now reinforces the symmetry axis.

In Experiment 3, we took the $120^{\circ}$ shape and presented it at various deviations away from the horizontal, the orientation where error rates for this shape were highest in Experiment 2. We did this to investigate further the role of perceived elongation. We replicated our previous finding that errors are highest at horizontal symmetry axis orientations. One interpretation of these results is that symmetry and elongation compete for responses. The results for Experiment 3 support the competition hypothesis: response direction in every case was an accommodation between factors like gravity and symmetry. When these factors reinforce, they strongly determine pointing. When they compete, pointing is an accommodation between them and responses align in-between but closer to the stronger influence.

One of the surprising results of our study was that global geometric factors such as symmetry and elongation overrode more local effects such as the proximity between dots. An axis is a prominent feature of a single object and can help localize its center. Our results suggest that this is also the case for multiple objects. The visual system effectively treats our displays as if they were a single object rather than a collection of disparate parts.

To reiterate, center estimation for multiple objects of the sort we use here is useful in a variety of everyday situations. It allows for the balancing of objects on a surface, the grasping and manipulation of single objects with irregular mass distributions, and the tracking of group location. Our results demonstrate that at least four factors must be taken into consideration when determining the perceptual center for these sorts of patterns.

\section{CONCLUSION}

Estimation of center of mass in triangular dot patterns can be accounted for by four factors: size ratio, gravity, symmetry and elongation. When the size of a dot is at least double that of the others, its "perceptual mass" attracts responses. When all dots are the same size, responses align along axes of symmetry and toward the gravitational down.
The presence of elongation also has an influence. Error rates increase the greater the length of an elongation axis. The effect is enhanced when elongation and symmetry are perpendicular and thus compete for responses. The orientation of these axes makes a difference. Center estimation is best for vertical symmetry and horizontal elongation and worst for horizontal symmetry and vertical elongation. Center estimation in these patterns is affected most by global configural properties, those that apply over the entire display.

\section{ACKNOWLEDGEMENT}

The authors would like to acknowledge Michael Alfieri who scheduled and collected data from many of the participants.

\section{REFERENCES}

[1] Beghi L, Vicario G, Zanforlin M. The perceptual centre of visual configurations. Atti e Memorie della Accademia Patavina di Scienze, Lettere ed Arti: Parte III. Classe di Scienze Morali, Lettere ed Arti 1983; 95: 133-148.

[2] Yakimoff N, Bocheva N, Mitrani L. Perceiving the center of irregular contour quadrangles. Spat Vis 1990; 5: 51-57.

[3] Bingham GP, Muchisky MM. Center of mass perception and inertial frames of reference. Percept Psychophys 1993a; 54: 617-632.

[4] Davi M. A model for identifying the perceptual centre of polygons. Perception 1994; 23: 1085-1095.

[5] Proffitt DR, Thomas MA, O'Brien RG. The roles of contour and luminance distribution in determining perceived centers within shapes. Percept Psychophys 1983; 33: 63-71.

[6] Davi M, Doyle, MAT, Proffitt, DR. The role of symmetry in determining perceived centers within shapes. Percept Psychophys 1992; 52: 151-160.

[7] Bingham G P, Muchisky MM. Center of mass perception: Perturbation of symmetry. Percept Psychophys 1993b; 54: 633-639.

[8] Baud-Bovy G, Soechting J. Visual localization of the center of mass of compact, asymmetric, two-dimensional shapes. J Exp Psychol Hum Percept Perform 2001; 27: 692-706.

[9] Gatti A. "Sull'apprezzamento del centro in alcune figure piane geometriche". Riv Psicol Soc Arch Ital Psicol Gen 1924; 3: 227244.

[10] Davi M. "Il centro percettivo di figure piane". Giornale Italiano di Psicologia 1989; XVI: 45-60.

[11] Friedenberg J, Liby B. Perception of two-body center of mass. Percep Psychophys 2002; 64: 531-539.

[12] Kubovy M, Wagemans J. Grouping by proximity and multistability in dot lattices: A quantitative gestalt theory. Psychol Sci 1995; 6 : 225-234.

[13] Wenderoth P. The salience of vertical symmetry. Percept 1994; 23: 221-236. 